\title{
STABILIZATION OF SCHRÖDINGER EQUATION IN EXTERIOR DOMAINS*
}

\author{
LassaAd Aloui $^{1,2}$ AND Moez Khenissi ${ }^{2,3}$
}

\begin{abstract}
We prove uniform local energy estimates of solutions to the damped Schrödinger equation in exterior domains under the hypothesis of the Exterior Geometric Control. These estimates are derived from the resolvent properties.
\end{abstract}

Mathematics Subject Classification. 47A10, 35A27, 35A21, 35A25.

Received February 4, 2005. Revised November 6, 2005.

Published online June 20, 2007.

\section{INTRODUCTION}

Let $\Omega$ be the exterior domain of a compact set in $\mathbb{R}^{d}(d \geq 2)$ with smooth boundary $\partial \Omega$. Let $R>0$ such that $\partial \Omega \subset B_{R}=\left\{x \in \mathbb{R}^{d} ; \quad|x|<R\right\}$. For $r>R$, we denote $\Omega_{r}=\Omega \cap B_{r}$.

We consider the following Schrödinger equation on $\Omega$

$$
\begin{cases}i \partial_{t} u-\Delta u=0 & \text { in } \quad \mathbb{R} \times \Omega \\ u(0, .)=f \in L^{2}(\Omega) & \text { in } \quad \Omega \\ u / \mathbb{R} \times \partial \Omega=0 & \end{cases}
$$

It is well known that the equation $(0.1)$ has a unique global solution $u \in C\left(\mathbb{R}, L^{2}(\Omega)\right)$. Moreover, the total energy $E(t)$ of the solution is conserved i.e.

$$
E(t):=\|u(t, .)\|_{L^{2}(\Omega)}=\|f\|_{L^{2}(\Omega)}, \quad \forall t \in \mathbb{R} .
$$

In the free case $\left(\Omega=\mathbb{R}^{d}\right)$, we have the following dispersive property

$$
\|u(t, .)\|_{L^{\infty}} \leq \frac{c}{t^{d / 2}}\|f\|_{L^{1}}, \quad \forall t>0
$$

Keywords and phrases. Cut-off resolvent, local energy decay, stabilization.

* The work of the LAMSIN's researchers is supported by the Tunisian Ministry for Scientific Research and Technology, within the LAB-STI 02 programme.

1 Département de Mathématiques, Faculté des Sciences de Bizerte, Tunisia; lassaad.aloui@fsg.rnu.tn

2 LAMSIN, Tunis, Tunisia.

3 Département de Mathématiques, Faculté des Sciences de Monastir, 5019 Monastir, Tunisia; moez.khenissi@fsg.rnu.tn

(c) EDP Sciences, SMAI 2007 
from which we deduce the uniform decay (polynomial) of the local energy:

$$
E_{r}(t):=\|u(t, .)\|_{L^{2}\left(B_{r}\right)} \leq \frac{c}{t^{d / 2}}\|f\|_{L^{2}}, \quad \forall t>0
$$

for all $f \in L^{2}$ supported in $B_{R}$.

When $\Omega$ is a non-trapping domain ${ }^{1}$, Vainberg [12-15] proved that the local energy of solutions of $(0.1)$ decay like $\frac{1}{t}$ as $t \rightarrow+\infty$ if $d$ is even and decay like $\frac{1}{t^{3 / 2}}$ as $t \rightarrow+\infty$ if $d$ is odd. Translating his resolvent estimates for $\left(\lambda^{2}+\Delta\right)^{-1}$ into estimates for $(i \tau+\Delta)^{-1}$, Tsutsumi [11] improved this result and showed that the decay rate is $O\left(\frac{1}{t^{d / 2}}\right)$.

The study of the resolvent $\left(\lambda^{2}+\Delta\right)^{-1}$ often enables to establish the local energy decay of solutions of the wave equations. More precisely, the decay is uniform if and only if the resolvent $\left(\lambda^{2}+\Delta\right)^{-1}$ is bounded as an operator from $L_{\text {comp }}^{2}(\Omega)$ to $L_{l o c}^{2}(\Omega)$ on a strip of the shape $\left\{\operatorname{Im} \lambda<c_{1},|\lambda|>c_{2} ; c_{1}, c_{2}>0\right\}$. For more details, see $[16]$.

It is known that, in the case of non-trapping geometries, $\left(\lambda^{2}+\Delta\right)^{-1}$ is bounded on the $\operatorname{set}\{\operatorname{Im} \lambda<$ $\left.c_{1} \log (|\operatorname{Re} \lambda|),|\lambda|>c_{2}\right\}$ (see [6]). This implies the uniform local energy decay of solutions of the wave equations.

In the trapping case, Ralston [8] shows that there is no uniform decay rate for the wave equation. Furthermore he gives an example with a sequence of poles of $\left(\lambda^{2}+\Delta\right)^{-1}$ converging exponentially to the real axis.

Finally, we would like to note the large litterature investigating the spectral properties of various perturbations of $-\Delta$ (like metric perturbations, Schrödinger operator with potential $-\Delta+V$, or combination of such problems) and giving local energy decay results for Schrödinger and wave equations (see for example, $[4,9]$ ).

Now we come back to the principal goal of the present paper: stabilization of equation (0.1). Roughly speaking, in the case of a trapping obstacle, this problem consists in acting on the system in order to obtain a uniform decay of the local energy $(0.1)$.

For the wave equation in odd space dimension, Aloui and Khenissi [1] introduce a damping term of type $a(x) \partial_{t} u \quad(a \geq 0)$; assuming then the E.G.C. (Exterior Geometric Control) condition on the couple ( $\omega=$ $\{a(x)>0\}, T)$ for some $T>0$, they prove a stabilization result. In the same framework, Khenissi [5] proves that the resolvent $\left(\lambda^{2}+\Delta-i a(x) \lambda\right)^{-1}$ is bounded from $L_{\text {comp }}^{2}(\Omega)$ to $L_{\text {loc }}^{2}(\Omega)$ in a strip of the shape $\left\{\operatorname{Im} \lambda<c_{1},|\lambda|>c_{2}\right.$, with $\left.c_{1}, c_{2}>0\right\}$ and he thus deduces the uniform decay of the local energy in any space dimension.

In this paper, we consider the following stabilization problem of the Schrödinger equation

$$
\begin{cases}i \partial_{t} u-\Delta u+i a(x) u=0 & \text { in } \mathbb{R} \times \Omega \\ u(0, .)=f & \text { in } \Omega \\ u / \mathbb{R} \times \partial \Omega=0 & \end{cases}
$$

where $a \in C_{0}^{\infty}(\Omega)$ is non-negative and $f \in L_{R}^{2}(\Omega)=\left\{g \in L^{2}(\Omega) / \operatorname{Supp} g \subset B(0, R)\right\}$.

Consider the operator $A_{a}=-i \Delta-a I$ defined on $L^{2}(\Omega)$ with domain

$$
D\left(A_{a}\right)=\left\{f \in L^{2}(\Omega) / \Delta f \in L^{2}(\Omega) \text { and } f_{/ \partial \Omega}=0\right\}
$$

One can easily show that $A_{a}$ is maximal dissipative (same proof as [1], Prop. 4.1); according then to the Hille-Yoshida theorem, it generates a semi group of contractions $U(t)$ such that for $f \in L^{2}(\Omega), U(t) f \in$ $C\left(\left[0,+\infty\left[, L^{2}(\Omega)\right)\right.\right.$ is the unique solution of $(0.5)$.

\footnotetext{
${ }^{1}$ Starting at a point $x$ in $\bar{\Omega}_{R}$ draw a ray in some direction $\xi$ and reflect it according to the classical law of geometric optics every time it hits the obstacle. Denote by $l(x, \xi)$ the total length (possibly infinity) of that ray within $\bar{\Omega}_{R}$ and by $l(R)$ the supermum of $l(x, \xi)$ for all such $x, \xi$. If $l(R)<\infty$ then $\Omega$ is said to be non-trapping.
} 
On the other hand, it is well known that for Schrödinger type equations, the speed of propagation is infinite, (contrary to that of the waves); for it, we go to weaken the E.G.C. condition used for waves [1] in the following way:

Definition 1 (E.G.C.). We say that the subset $\omega$ of $\Omega$ satisfies the Exterior Geometric Control (E.G.C.) if any trapped ray $^{2}$ meets $\omega$.

For $\tau \in\{\operatorname{Re} \tau<0, \operatorname{Im} \sqrt{i \tau} \geq 0\}$,we denote by $R(\tau) f=(-i \tau-\Delta+i a)^{-1} f$ the unique finite energy solution of the following system

$$
\left\{\begin{array}{l}
(-i \tau-\Delta+i a) v=f \text { in } \Omega, \\
v_{/ \partial \Omega}=0 \\
v \text { outgoing. }
\end{array}\right.
$$

$R(\tau)$ is known as the outgoing resolvent associated to the problem (0.5). It is easy to see that

$$
R(\tau) f=i \int_{0}^{+\infty} \mathrm{e}^{\tau t} u(t) \mathrm{d} t
$$

with $u(t)$ solution of (0.5). It is also clear that the relation (0.6) defines a family of bounded operators from $L^{2}(\Omega)$ to $L^{2}(\Omega)$, holomorphic on $\{\operatorname{Re} \tau<0\}$.

Let $\chi \in C_{0}^{\infty}\left(\mathbb{R}^{d}\right), \chi=1$ on $B_{r}, \chi=0$ on $\mathbb{R}^{d} \backslash B_{r^{\prime}}$ where $r^{\prime}>r>R$. The cut-off resolvent

$$
R_{\chi}(\tau)=\chi R(\tau) \chi
$$

considered as operator from $L^{2}(\Omega)$ to $L^{2}(\Omega)$, holomorphic on $\{\operatorname{Re} \tau<0\}$ extends to a meromorphic operator on the logarithmic plane in even space dimension and on the $\tau^{2}$-plane (the two sheeted Riemann surface for $\sqrt{i \tau}$, see $[17]$ ) in odd space dimension. And we have the following theorem.

Theorem 1. Suppose that $\omega=\{x \in \Omega, a(x)>0\}$ satisfies the E.G.C., then there exist positive constants $\alpha$ and $\beta$ such that $R_{\chi}(\tau)$ has no pole in the region

$$
\Lambda_{\alpha, \beta}=\{\tau \in \mathbb{C} \quad / \quad \operatorname{Re} \tau \leq \alpha ;|\operatorname{Im} \tau| \geq \beta\}
$$

Furthermore, there exists $c>0$ such that for any $\tau \in \Lambda_{\alpha, \beta}$

$$
\left\|R_{\chi}(\tau)\right\|_{L^{2}(\Omega) \rightarrow L^{2}(\Omega)} \leq c .
$$

From this, we deduce the following stabilization result:

Theorem 2. Under the hypotheses of the Theorem 1, there exists $c>0$ such that for all $f \in L_{r}^{2}(\Omega)(r>R)$

$$
\|u(t, .)\|_{L^{2}\left(\Omega_{r}\right)} \leq \frac{c}{t^{d / 2}}\|f\|_{L^{2}(\Omega)}, \forall t>1
$$

with $u$ solution of $(0.5)$.

Remark 1. For the proof of Theorem 1, we choose $R$ large enough (in particular Supp $\left.a(x) \subset B_{R}\right)$ but the results remain true for all $R$ such that $\partial \Omega \subset B_{R}$.

This paper is organized as follows.

Section 1 is devoted to the proof of Theorem 1. First we verify that the behavior of the resolvent $R(\tau)$ near 0 (low frequencies) is like the free case. The rest of the proof is inspired from [3]; it consists on a contradiction argument and is essentially based on properties of semiclassical measures. Finally, in the second section, using the results of Theorem 1 and classical arguments (see $[5,7,11]$ ), we obtain the desired energy decay of Theorem 2.

\footnotetext{
${ }^{2} \mathrm{~A}$ trapped ray is a ray which can not leave $B_{R}$.
} 


\section{Cut-off Resolvent}

We denote by $\tilde{\mathbb{C}}$ the logarithmic plane in even space dimension and the $\tau^{2}$-plane in odd dimension. For two Banach spaces $X$ and $Y$ we denote by $\operatorname{Hom}(X, Y)$ the Banach space consisting of all bounded linear operators from $X$ to $Y$.

First, for $r^{\prime}>r>R$, we prove that the resolvent $R(\tau)=(-i \tau-\Delta+i a)^{-1}$, defined as a $\operatorname{Hom}\left(L_{r}^{2}(\Omega), L^{2}\left(\Omega_{r^{\prime}}\right)\right)$ valued holomorphic function with respect to $\tau \in \tilde{\mathbb{C}} \cap\{\operatorname{Re} \tau<0\}$, admits a meromorphic extension to $\tilde{\mathbb{C}}$.

As in [11], we consider $\alpha_{1}, \alpha_{2}$ the following step functions

$$
\alpha_{1}(x)=\left\{\begin{array}{ll}
0 & \text { if }|x|>R+\frac{1}{2} \\
1 & \text { otherwise, }
\end{array} \quad \alpha_{2}(x)=1-\alpha_{1}(x)\right.
$$

and $\beta_{1}(x), \beta_{2}(x)$ two real valued $C^{\infty}$ functions such that

$$
\begin{aligned}
& \beta_{1}(x)= \begin{cases}0 & \text { if }|x|>R+1 \\
1 & \text { if }|x|<R+\frac{2}{3}\end{cases} \\
& \beta_{2}(x)= \begin{cases}0 & \text { if }|x|<R \\
1 & \text { if }|x|>R+\frac{1}{3}\end{cases}
\end{aligned}
$$

We denote $L_{\tau_{0}}$ the operator which maps a function $f \in L_{r}^{2}(\Omega)$ into the solution $u(x) \in H^{2}\left(\Omega_{r}\right)$ of the problem

$$
\left\{\begin{array}{l}
\left(-\Delta-i \tau_{0}\right) u=f \quad x \in \Omega_{r} \\
u / \partial \Omega_{r}=0
\end{array}\right.
$$

where $r>R+1$ and $\tau_{0}$ chosen in $\tilde{\mathbb{C}}$ such that the problem (1.1) is well posed. We define the operator $G_{\tau}$ from $L_{r}^{2}(\Omega)$ to $H^{2}\left(\Omega_{r^{\prime}}\right)$ by

$$
G_{\tau} g=\beta_{1}(x) L_{\tau_{0}}\left(\alpha_{1}(x) g\right)+\beta_{2}(x) R_{0}(\tau) \alpha_{2}(x) g
$$

for all $g \in L_{r}^{2}(\Omega)$, with $r^{\prime}>r$ and $R_{0}(\tau)$ is the analytic extension of $(-\Delta-i \tau)^{-1}$ on $\tilde{\mathbb{C}}$ for $\Omega=\mathbb{R}^{d} . R_{0}(\tau)$ maps a function $f \in L_{r}^{2}\left(\mathbb{R}^{d}\right)$ into the solution $w \in H^{2}\left(\Omega_{r^{\prime}}\right)$ of the following problem

$$
\left\{\begin{array}{l}
(-\Delta-i \tau) w=f, \quad x \in \mathbb{R}^{d} \\
w \text { outgoing. }
\end{array}\right.
$$

Note that $G_{\tau}$ is a bounded operator from $L_{r}^{2}(\Omega)$ to $H^{2}\left(\Omega_{r^{\prime}}\right)$ and holomorphic in $\tilde{\mathbb{C}}$, so $a G_{\tau}$ is a compact operator from $L_{r}^{2}(\Omega)$ to $L^{2}\left(\Omega_{r^{\prime}}\right)$ for all $\tau \in \tilde{\mathbb{C}}$.

Consider the operator

$$
\begin{aligned}
S_{a}(\tau) & =(-\Delta-i \tau+i a) G_{\tau}-I \\
& =(-\Delta-i \tau) G_{\tau}-I+i a G_{\tau} \\
& =S(\tau)+i a G_{\tau}
\end{aligned}
$$

It is known, from [11], that $S(\tau)$ is a compact operator from $L_{r}^{2}(\Omega)$ to $L_{r}^{2}(\Omega)$ for all $\tau \in \tilde{\mathbb{C}}$. Then we deduce that $S_{a}(\tau)$ is a compact operator from $L_{r}^{2}(\Omega)$ to $L_{r}^{2}(\Omega)$ for all $\tau \in \tilde{\mathbb{C}}$.

Let us prove that the operator $S_{a}(\tau)+I$ has a bounded inverse. From the Fredholm theory, it is sufficient to show that $S_{a}(0)+I$ is one to one.

Let $g \in L_{r}^{2}(\Omega)$ such that

$$
\left(S_{a}(0)+I\right) g=0
$$


We have

$$
\left(S_{a}(0)+I\right)=(-\Delta+i a) G_{0}
$$

and

$$
G_{0} g \underset{|x| \rightarrow \infty}{\longrightarrow} 0
$$

So $u \equiv G_{0} g$ satisfies

$$
\left\{\begin{array}{l}
(-\Delta+i a) u=0, \quad x \in \Omega \\
u_{/ \partial \Omega}=0, u \underset{|x| \rightarrow+\infty}{\longrightarrow} 0 .
\end{array}\right.
$$

Multiplying the equation in (1.3) by $\bar{u}$, integrating over $\Omega$ and taking the real part, we deduce that the null function is the unique solution of this system. From [11], $G_{0}$ is one to one, then $g=0$.

Now, according to the Fredholm's theory [10], Theorem VI.14, and (1.2), $\left(I+S_{a}(\tau)\right)$ has a meromorphic inverse and

$$
R(\tau)=(-\Delta-i \tau+i a)^{-1}=G_{\tau}\left(I+S_{a}(\tau)\right)^{-1} .
$$

We deduce that $R(\tau)$ has a meromorphic extension on $\tilde{\mathbb{C}}$.

\subsection{Low frequencies}

We are going to show that $R(\tau)$ has the same behavior as the free resolvent $R_{0}(\tau)$ near $\tau=0$. More precisely we have

Lemma 1. Let $d \geq 2, r^{\prime}>r>R$. There exists $\varepsilon>0$ such that

(1) If $d$ is odd, the operator $R(\tau)$ has no poles in the domain $\{\tau: \tau \in \tilde{\mathbb{C}} /|\tau|<\varepsilon\}$ and can be represented in the form

$$
R(\tau)=B_{1}(\tau)+\tau^{\frac{d-2}{2}} B_{2}(\tau)
$$

where $B_{1}(\tau)$ and $B_{2}(\tau)$ are a $\operatorname{Hom}\left(L_{r}^{2}(\Omega), H^{2}\left(\Omega_{r^{\prime}}\right)\right)$-valued holomorphic function.

(2) If $d$ is even, the operator $R(\tau)$ has no poles in the domain $\{\tau \in \tilde{\mathbb{C}} /|\tau|<\varepsilon ;-\pi<\arg \tau<3 \pi\}$ and can be represented in the form

$$
R(\tau)=B_{3}(\tau)+\tau^{\frac{d-2}{2}} \log \sqrt{i \tau} B_{4}+\tau^{\frac{d-2}{2}} B_{5}(\tau)
$$

where $B_{3}(\tau)$ is a $\operatorname{Hom}\left(L_{r}^{2}(\Omega), H^{2}\left(\Omega_{r^{\prime}}\right)\right)$-valued holomorphic function on $\{\tau \in \tilde{\mathbb{C}} /|\tau|<\varepsilon\}, B_{4}$ is a bounded operator from $L_{r}^{2}(\Omega)$ to $H^{2}\left(\Omega_{r^{\prime}}\right)$, and $B_{5}(\tau)$ is a $\operatorname{Hom}\left(L_{r}^{2}(\Omega), H^{2}\left(\Omega_{r^{\prime}}\right)\right)$-valued bounded continuous function.

Proof. According to the previous section, $I+S_{a}(0)$ is invertible, then we have

$$
\begin{aligned}
\left(I+S_{a}(\tau)\right)^{-1} & =\left(I+S_{a}(0)+S_{a}(\tau)-S_{a}(0)\right)^{-1} \\
& =\left(I+\left(I+S_{a}(0)\right)^{-1}\left(S_{a}(\tau)-S_{a}(0)\right)\right)^{-1}\left(I+S_{a}(0)\right)^{-1} .
\end{aligned}
$$

So for $\tau$ small enough

$$
\left(I+S_{a}(\tau)\right)^{-1}=\left(\sum_{k \geq 0}(-1)^{k}\left(\left(I+S_{a}(0)\right)^{-1}\left(S_{a}(\tau)-S_{a}(0)\right)\right)^{k}\right)\left(I+S_{a}(0)\right)^{-1} .
$$

Since the expansion of the types (1.4), (1.5) holds for $R_{0}(\tau)$, we obtain the expansion of the types (1.4), (1.5) for $G_{\tau}$ and $S_{a}(\tau)$. From (1.2) we have

$$
R(\tau)=G_{\tau}\left(I+S_{a}(\tau)\right)^{-1}
$$

thus $R(\tau)$ satisfies (1.4) and (1.5). 


\subsection{High frequencies}

In this section, we will prove the estimate of Theorem 1 by contradiction. We refer to [3] for the definition and properties of semiclassical measures.

Since $R_{\chi}(\tau)$ considered as operator in $L^{2}(\Omega)$, is meromorphic in $\tilde{\mathbb{C}}$, it is sufficient to prove the estimate of Theorem 1 for $\tau$ belonging to the resolvent set and $\Lambda_{\alpha, \beta}[5]$.

By contradiction, we may assume the existence of two sequences $\left(f_{n}\right) \in L_{r}^{2}(\Omega)$ and $\left(\tau_{n}\right) \in \mathbb{C}$ such that $\left|\operatorname{Im} \tau_{n}\right| \geq n, 0<\operatorname{Re} \tau_{n}<\frac{1}{n}$ and

$$
\left\|R_{\chi}\left(\tau_{n}\right) f_{n}\right\|_{L^{2}(\Omega)} \geq n\left\|f_{n}\right\|_{L^{2}(\Omega)} .
$$

We denote $u_{n}=R\left(\tau_{n}\right) f_{n}$, and we normalize it by $\left\|\chi u_{n}\right\|_{L^{2}(\Omega)}$. This gives

$$
\begin{aligned}
& \left\{\begin{array}{l}
(-i \tau-\Delta+i a) u_{n}=f_{n} \text { in } \Omega \\
u_{n / \partial \Omega}=0 \\
u_{n} \text { outgoing }
\end{array}\right. \\
& \left\|\chi u_{n}\right\|_{L^{2}(\Omega)}=1,\left\|f_{n}\right\|_{L^{2}} \longrightarrow 0
\end{aligned}
$$

We distinguish two cases:

1st case (where $A_{a}$ is coercive). There exists a subsequence of $\left(\tau_{n}\right)$, still denoted by $\left(\tau_{n}\right)$, such that

$$
\operatorname{Im} \tau_{n} \geq n
$$

Set $h_{n}=\frac{1}{\sqrt{\operatorname{Im} \tau_{n}}} ;$ we have

$$
\left[-h_{n}^{2} \Delta+1+i h_{n}^{2}\left(a(x)-\operatorname{Re} \tau_{n}\right)\right] u_{n}=h_{n}^{2} f_{n}
$$

We multiply the equation (1.8) by $\bar{u}_{n}$ and integrate by parts, we obtain

$$
h_{n}^{2}\left\|\nabla u_{n}\right\|_{L^{2}}^{2}+\left\|u_{n}\right\|_{L^{2}}^{2}=h_{n}^{2} \operatorname{Re}\left(\int f_{n} \bar{u}_{n}\right) \underset{n \rightarrow \infty}{\longrightarrow} 0
$$

which contradicts

$$
\left\|\chi u_{n}\right\|_{L^{2}(\Omega)}=1
$$

2nd case. There exists a subsequence of $\left(\tau_{n}\right)$, still denoted by $\left(\tau_{n}\right)$, such that

$$
\operatorname{Im} \tau_{n} \leq-n
$$

Set $h_{n}=\frac{1}{\sqrt{-\operatorname{Im} \tau_{n}}}$, then $u_{n}$ satisfy

$$
\left[-h_{n}^{2} \Delta-1+i h_{n}^{2}\left(a(x)-\operatorname{Re} \tau_{n}\right)\right] u_{n}=h_{n}^{2} f_{n}
$$

We study now the sequence $\left(u_{n}\right)_{n \in \mathbb{N}}$.

Lemma 2. The sequence $\left(u_{n}\right)_{n \in \mathbb{N}}$ is bounded in $L_{l o c}^{2}(\Omega)$.

Proof. Let $\varphi \in C_{0}^{\infty}$ equal to 1 near $\partial \Omega$ and in a neighborhood of the support of $a(x)$ and such that Supp $(\varphi) \subset B_{R}$ (see Rem. 1). 
Let $\omega_{n}=(1-\varphi) u_{n}$. We have

$$
\left(-h_{n}^{2} \Delta-1-i h_{n}^{2} \operatorname{Re} \tau_{n}\right) \omega_{n}=h_{n}^{2} f_{n}(1-\varphi)-[\Delta, \varphi] h_{n}^{2} u_{n} .
$$

This equation has the following form

$$
\left(-h_{n}^{2} \Delta-z_{n}\right) \omega_{n}=g_{n} \text { in } \mathbb{R}^{d}
$$

where $z_{n}=1+i h_{n}^{2} \operatorname{Re} \tau_{n}$ and $g_{n}=h_{n}^{2} f_{n}(1-\varphi)-[\Delta, \varphi] h_{n}^{2} u_{n}$.

Hence

$$
\omega_{n}=R_{0}\left(h_{n}, z_{n}\right) g_{n}
$$

where $R_{0}(h, z)=\left(-h^{2} \Delta-z\right)^{-1}$ is the free resolvent of the semi-classical Laplace operator $-h^{2} \Delta$. Since Supp $g_{n} \subset B_{r},\left\|g_{n}\right\|_{L^{2}}$ is bounded (we use that $h_{n} \nabla u_{n}$ is bounded in $L^{2}$ near the support of $\nabla \varphi$ ). Then we deduce from [3] that $\omega_{n}$ is bounded in $L_{l o c}^{2}$. According to (1.9), $u_{n}$ is bounded in $L_{l o c}^{2}(\Omega)$.

Now, we are able to associate to the sequence $\left(u_{n}\right)$ a semiclassical measure in $L_{l o c}^{2}(\Omega)$ (a positive Radon measure in Melrose's compressed bundle [3]) denoted by $\mu$. This measure satisfies the two following microlocal properties.

Lemma 3. We have Supp $\mu \cap\{(x, \xi), x . \xi \leq 0,|x| \geq R\}=\emptyset$.

This result is a consequence of (1.11) and the Proposition 2.2 of [3] (see also [5]) which is a microlocal interpretation of the outgoing behavior of the free cut-off resolvent.

Moreover, recalling that $\omega=\{x \in \Omega / a(x)>0\}$, we have

Lemma 4. Supp $\mu \cap T^{*} \omega=\emptyset$.

Proof. We multiply (1.10) by $\bar{u}_{n}$, integrate over $\Omega_{r}$ and take the imaginary part. We obtain

$$
\int_{\Omega_{R}} a(x)\left|u_{n}\right|^{2} \mathrm{~d} x=\operatorname{Re} \tau_{n} \int_{\Omega_{r}}\left|u_{n}\right|^{2} \mathrm{~d} x+\operatorname{Im}\left(\int_{\Omega_{R}} f_{n} \bar{u}_{n}\right) \mathrm{d} x+\operatorname{Im} \int_{|x|=r} \frac{\partial u_{n}}{\partial r} \bar{u}_{n} \mathrm{~d} \sigma .
$$

Now we use the following

Proposition 1 (see Burq [2]). For any $r>R>0$, there exist $C_{1}, C_{2}, \eta, \lambda_{0}>0$ such that for any outgoing solution of $(\Delta+z) u=0$ outside a ball $B\left(0, R_{1}\right)$ and any $z \in \mathbb{C}:|\operatorname{Im} \sqrt{z}| \leq 1,|\sqrt{z}| \geq \lambda_{0}$, we have

$$
\begin{aligned}
-\operatorname{Im} \int_{|x|=r} \frac{\partial u}{\partial r} \bar{u} \mathrm{~d} \sigma \geq & |\sqrt{z}| C_{1} \int_{|x|=r}|u|^{2}+\left|\sqrt{z}^{-1} \nabla u\right|^{2} \mathrm{~d} \sigma \\
& -C_{2} \mathrm{e}^{-\eta|\sqrt{z}|} \int_{|x|=R}|u|^{2}+\left|\sqrt{z}^{-1} \nabla u\right|^{2} \mathrm{~d} \sigma .
\end{aligned}
$$

According to Lemma 2 and $1.10, h_{n}^{2} \Delta u_{n}$ is bounded in $L_{l o c}^{2}(\Omega)$, then $h_{n}^{4} \int_{|x|=R}\left|u_{n}\right|^{2} \mathrm{~d} \sigma, \int_{|x|=R}\left|h_{n}^{2} \nabla u\right|^{2} \mathrm{~d} \sigma$ are bounded. Since $\operatorname{Im} \sqrt{i \tau_{n}} \rightarrow 0$ and $h_{n}^{-4} \mathrm{e}^{-\eta\left|\sqrt{\tau_{n}}\right|} \rightarrow 0$, this shows, thanks to Proposition 1 , that

$$
\lim \inf _{n \rightarrow+\infty} \operatorname{Im} \int_{|x|=r} \frac{\partial u_{n}}{\partial r} \bar{u}_{n} \mathrm{~d} \sigma \leq 0,
$$

thus, we can deduce that

$$
\int_{\Omega_{R}} a(x)\left|u_{n}\right|^{2} \mathrm{~d} x \longrightarrow 0
$$

which yields $\mu \equiv 0$ on $\omega$. 
Here we finish the proof of Theorem 1. We deduce, from equation (1.10), that the sequence $\left(u_{n}\right)$ is $h_{n^{-}}$ oscillating (see [3] for more details). Hence

$$
\left\langle\mu, \chi(x) \times 1_{\xi}\right\rangle=\lim _{n \longrightarrow+\infty}\left\|\chi u_{n}\right\|^{2}=1 \neq 0 .
$$

And we will get the announced contradiction, if we show that $\mu \equiv 0$. We recall from [3], Propositions 4.4 and 4.6, that the measure $\mu$ is invariant along the generalized bicharacteristic flow associated to the symbol $|\xi|^{2}-1$.

Let $q=(x, \xi) \in T_{b}^{*} \Omega$ (Melrose's compressed cotangent bundle (see [3], Sect. 4.1)) and $\gamma$ be a generalized bicharacteristic curve issued from $q$. We have to consider two cases:

1st case. $\gamma$ is a trapped ray ${ }^{3}$. By the E.G.C. assumption, $\gamma$ meets the stabilization region $T^{*} \omega$ and by Lemma 2 $\mu=0$ on $T^{*} \omega$. Applying then the measure propagation result of Burq [3], we conclude that $\mu=0$ near $q$.

2nd case. $\gamma$ is a non-trapped ray. In this case $\gamma$ meets the incoming region $\{x . \xi \leq 0,|x| \geq R\}$ where the measure $\mu$ is null (see Lem. 3). Using again the measure propagation, we deduce that $\mu=0$ near $q$.

Thus we conclude that $\mu$ is identically null, which ends the proof.

\section{DeCAy OF THE LOCAL ENERGY}

In this section we give the proof of Theorem 2.

Our strategy is to recover $u(t)$ by inverting the Laplace transform and to shift the contour of integration into the halfplan $\operatorname{Re} \tau>0$, using Cauchy's theorem. In this way, the results of the first section provide the local energy decay.

Remark 2. In applying the Cauchy integral theorem some convergence problems have to be analyzed to justify the shift contour. This is possible for initial data with higher regularity only. But by continuity and density arguments, the resulting identities still hold for initial data with finite energy.

We recall that

$$
R(\tau) f=i \int_{0}^{+\infty} \mathrm{e}^{\tau t} u(t) \mathrm{d} t, \quad \operatorname{Re} \tau<0,
$$

and for all $t \geq 0$ the inverse Laplace transform

$$
u(t)=-(2 \pi)^{-1} \int_{\operatorname{Re} \tau=-\delta} \mathrm{e}^{-t \tau} R(\tau) f \mathrm{~d} \tau, \forall \delta>0 .
$$

Here the integral is to be understood as

$$
\lim _{M \rightarrow \infty} \int_{-i M-\delta}^{i M-\delta} \mathrm{e}^{-t \tau} R(\tau) f \mathrm{~d} \tau
$$

where the convergence in $L^{2}(\Omega)$ is uniform for $t$ in bounded intervals. Unfortunately the real part of $\tau$ seems to have the wrong sign to derive good estimates of the solution out of (2.1). The path of integration has to be moved. According to Theorem 1 and using the behavior of $R(\tau)$ at low frequencies, there exists $\alpha_{0}>0$ such that $\forall \varepsilon>0, R(\tau)$ is analytic in the region which is hatched in Figure 1. By $\Gamma_{\varepsilon}$ we denote the whole contour defined by $\Gamma_{\varepsilon}=\Gamma_{1 \varepsilon}^{+} \cup \Gamma_{1 \epsilon}^{-} \cup \Gamma_{2 \varepsilon}^{+} \cup \Gamma_{2 \epsilon}^{-}$where $\Gamma_{1 \varepsilon}^{ \pm}=\left\{\alpha_{0} \pm i y ; y>\varepsilon\right\}$ and $\Gamma_{2 \varepsilon}^{ \pm}=\left\{\left(1 \pm i \frac{\varepsilon}{\alpha_{0}}\right) s ; 0 \leq s \leq \alpha_{0}\right\}$.

\footnotetext{
${ }^{3}$ We identify here $\gamma$ to its projection in $\bar{\Omega}$, then it is also called ray.
} 


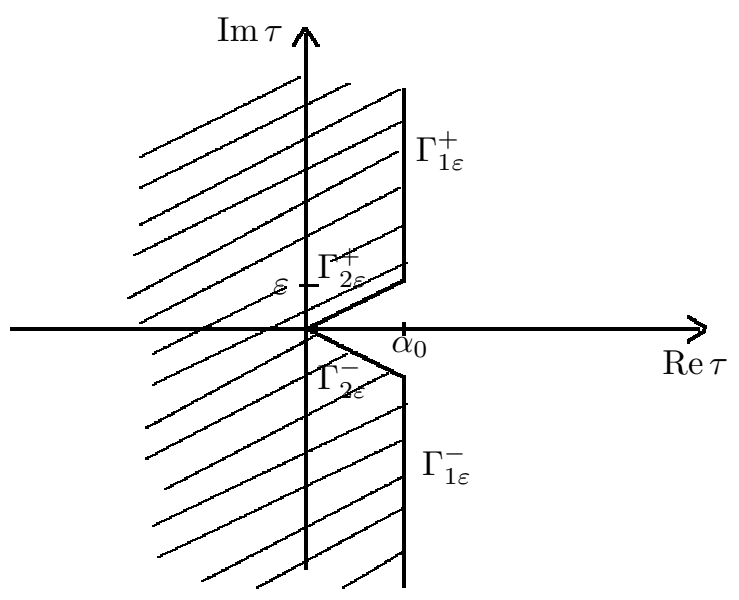

FiguRE 1. Free of poles region.

Due to (2.1) and the Cauchy Theorem and Remark 2, we can write

$$
\begin{gathered}
u(t)=-(2 \pi)^{-1} \int_{\Gamma_{\varepsilon}} \mathrm{e}^{-t \tau} R(\tau) f \mathrm{~d} \tau, \forall \varepsilon>0 \\
u(t)=-(2 \pi)^{-1} \mathrm{e}^{-\alpha_{0} t} \int_{-\infty}^{+\infty} \mathrm{e}^{-i t y} R\left(\alpha_{0}+i y\right) f \mathrm{~d} y \\
+(2 \pi)^{-1}\left(\lim _{\varepsilon \rightarrow 0}\left(\int_{\Gamma_{2 \varepsilon}^{-}} \mathrm{e}^{t \tau} R(\tau) f \mathrm{~d} \tau-\int_{\Gamma_{2 \epsilon}^{+}} \mathrm{e}^{t \tau} R(\tau) f \mathrm{~d} \tau\right)\right) \\
=W_{1}(t) f+W_{2}(t) f .
\end{gathered}
$$

From the resolvent equation, we get for $f \in D\left(A_{a}^{2}\right) \cap L_{r}^{2}(\Omega)$

$$
R_{\chi}(\tau) f=i \frac{1}{\tau} \chi\left(I+A_{a}\right) \chi+\frac{1}{\tau^{2}} R_{\chi}(\tau) A_{a}^{2} f .
$$

Since $\left\|R\left(\alpha_{0}+i y\right) f\right\|_{L^{2}\left(\Omega_{r}\right)}$ is bounded, we obtain as in [11] ([9])

$$
\left\|W_{1}(t) f\right\|_{L^{2}\left(\Omega_{r}\right)} \leq c \mathrm{e}^{-c t}\|f\|_{L^{2}(\Omega)}, \quad t \geq 1
$$

and Lemma 1 (see $[5,9,11])$ gives

$$
\left\|W_{2}(t) f\right\|_{L^{2}\left(\Omega_{r}\right)} \leq C t^{-d / 2}\|f\|_{L^{2}(\Omega)} \quad t \geq 1 .
$$

Finally, we obtain

$$
\|u(t)\|_{L_{r}^{2}(\Omega)} \leq C t^{-d / 2}\|f\|_{L^{2}(\Omega)}, \forall t \geq 1 .
$$

Remark 3. It should be remarked that the high-frequency behavior decides whether there is uniform local energy decay or not. But the explicit rate is calculated from the low-frequency asymptotic. 
Acknowledgements. The authors wish to express their thanks to the referees for helpful remarks and suggestions and for pointing out a mistake in the first version of this paper. The authors are also indebted to Professor B. Dehman for his valuable conversation and constant encouragement.

\section{REFERENCES}

[1] L. Aloui and M. Khenissi, Stabilisation de l'équation des ondes dans un domaine extérieur. Rev. Math. Iberoamericana 28 (2002) 1-16.

[2] N. Burq, Décroissance de l'énergie locale de l'équation des ondes pour le problème extérieur et absence de résonance au voisinage du réel. Act. Math. 1 (1998) 1-29.

[3] N. Burq, Semi-classical estimates for the resolvent in non trapping geometries. Int. Math. Res. Not. 5 (2002) $221-241$.

[4] A. Jensen and T. Kato, Spectral properties of Schrödinger operators and time decay of the wave functions. Duke Math. J. 46 (1979) 583-612.

[5] M. Khenissi, Équation des ondes amorties dans un domaine extérieur. Bull. Soc. Math. France 131 (2003) 211-228.

[6] R.B. Melrose and J. Sjostrand, Singularities of boundary value problems I. Comm. Pure Appl. Math. 31 (1978) 593-617.

[7] C.S. Morawetz, Decay for solution of the exterior problem for the wave equation. Comm. Pure Appl. Math. 28 (1975) $229-264$.

[8] J. Ralston, Solution of the wave equation with localized energy. Comm. Pure Appl. Math. 22 (1969) 807-823.

[9] J. Rauch, Local decay of scattering solutions of Schrödinger-type equation. Comm. Math. Phys. 61 (1978) 149-168.

[10] M. Reed and B. Simon, Methods of modern mathematical physics, Vol. I: Functional Analysis. New York, Academic Press (1972).

[11] Y. Tsutsumi, Local energy decay of solutions to the free Schrödinger equation in exterior domains. J. Fac. Sci. Univ. Tokyo, Sect. IA, Math. 31 (1984) 97-108.

[12] B. Vainberg, On the analytical properties of the resolvent for certain class of operator-pencils. Math. USSR-Sb. 6 (1968) 241-273.

[13] B. Vainberg, On the exterior elliptic problems polynomially depending on a spectral parameters, and asymptotic behaviour for large time of solutions of non stationary problems. Math. USSR-Sb. 21 (1973) 221-239.

[14] B. Vainberg, On the short wave asymptotic behaviour of solutions of stationary problems and asymptotic behaviour as $\mathrm{t} \longrightarrow+\infty$ of solutions of non-stationary problems. Russian Math. Surveys 30 (1975) 1-58.

[15] B. Vainberg, Asymptotic methods in equations of mathematical physics. Gordon and Breach, New York (1988).

[16] G. Vodev, On the uniform decay of the local energy. Serdica Math. J. 25 (1999) 191-206.

[17] H. Wilcox, Scattering Theory for the d'Alembert Equation in Exterior Domains. Lect. Notes Math. 442, Springer-Verlag (1975). 